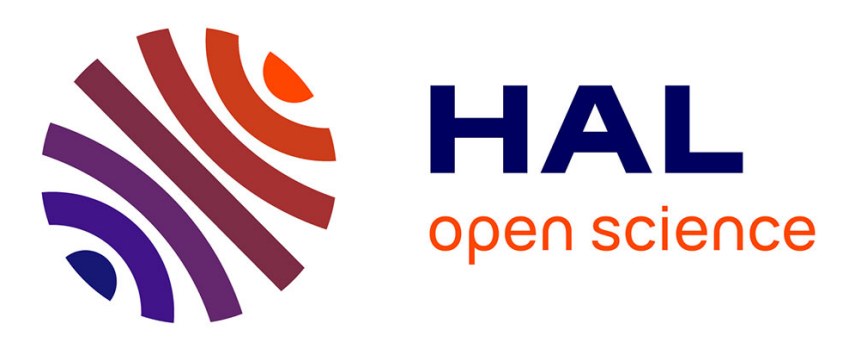

\title{
Probabilistic analysis of a one-dimensional soil consolidation problem
}

\author{
Youcef Houmadi, Ashraf Ahmed, Abdul-Hamid Soubra
}

\section{To cite this version:}

Youcef Houmadi, Ashraf Ahmed, Abdul-Hamid Soubra. Probabilistic analysis of a one-dimensional soil consolidation problem. Georisk: Assessment and Management of Risk for Engineered Systems and Geohazards, 2012, 6 (1), pp.36-49. 10.1080/17499518.2011.590090 . hal-01007204

\section{HAL Id: hal-01007204 \\ https://hal.science/hal-01007204}

Submitted on 11 Mar 2018

HAL is a multi-disciplinary open access archive for the deposit and dissemination of scientific research documents, whether they are published or not. The documents may come from teaching and research institutions in France or abroad, or from public or private research centers.
L'archive ouverte pluridisciplinaire HAL, est destinée au dépôt et à la diffusion de documents scientifiques de niveau recherche, publiés ou non, émanant des établissements d'enseignement et de recherche français ou étrangers, des laboratoires publics ou privés. 


\title{
Probabilistic analysis of a one-dimensional soil consolidation problem
}

\author{
Youcef Houmadi $^{\mathrm{a}, \mathrm{b}}$, Ashraf Ahmed ${ }^{\mathrm{b}}$ and Abdul-Hamid Soubra ${ }^{\mathrm{b} *}$ \\ ${ }^{a}$ Department of Civil Engineering, Saida University, Saida, Algeria; ${ }^{b}$ Departement of Civil Engineering, University of Nantes, \\ Saint-Nazaire, France
}

\begin{abstract}
In this paper, a probabilistic study of a one-dimensional soil consolidation problem has been carried out. The Collocation-based Stochastic Response Surface Method (CSRSM) was employed for the probabilistic analysis. The Young modulus $E$, the Poisson ratio $v$, the hydraulic conductivity $k_{h}$ and the uniform surcharge loading $q$ applied at the ground surface were considered as random variables. The probabilistic system responses considered in the analysis were the surface settlement and the consolidation time. Numerical simulations that make use of Biot theory were used for the computation of these system responses. A global sensitivity analysis based on Sobol indices was performed to identify the random variables that have the most contribution in the variability of the system responses. Also, a parametric study was undertaken to investigate the effect of the input geotechnical parameters and the statistical parameters of the random variables on the probability distribution functions of the system responses.
\end{abstract}

Keywords: consolidation; coupled Biot theory; probabilistic methods

\section{Introduction}

The effect of the uncertainties of the soil parameters and the surcharge loading on the surface elastic settlement or on the surface consolidation settlement has been investigated by several authors. Some investigators (Freeze 1977, Chang 1985, Hong 1992, Lee et al. 1992, Darrag and Tawil 1993, Bauer and Pula 2000) have considered the uncertain parameters as random variables. Others have modelled the uncertain parameters by random fields to take into account the soil spatial variability (Hwang and Witczak 1984, Nour et al. 2002, Badaoui et al. 2007, Griffiths and Fenton 2009, Huang et al. 2010). It should be emphasised here that most of these studies make use of Monte Carlo Simulation (MCS) methodology to compute the Probability Distribution Function (PDF) of the system response or the failure probability. This methodology is well-known to be very expensive because of the great number of calls of the deterministic model required for the probabilistic analyses. In this paper, an efficient probabilistic method called Collocation-based Stochastic Response Surface Method (CSRSM) was used. This method is based on the approximation of the system response by a Polynomial Chaos Expansion (PCE). It aims at replacing a complex deterministic model by a metamodel which is an approximate explicit analytical formula. This makes it easy to apply MCS methodology on the meta-model without the need to call the original deterministic numerical model. Also, CSRSM allows one to rigorously calculate the contribution of each random variable in the variability of the system response using Sobol indices (e.g. Sudret 2008, Mollon et al. 2011). This is very important because one can detect the uncertain variables that have the greatest contribution in the variability of the system response and thus, this leads to a reduction in the number of uncertain parameters that should be handled by the geotechnical engineer.

This paper is devoted to the probabilistic analysis of a coupled or an uncoupled one-dimensional (1D) consolidation problem of a single clayey layer using the CSRSM. The deterministic model is based on a Biot consolidation analysis using the finite difference code FLAC ${ }^{3 \mathrm{D}}$. The uncertain parameters were modelled by random variables. These are the Young modulus $E$, the Poisson ratio $v$, the hydraulic conductivity $k_{h}$ (related to the permeability coefficient $k$ by $k_{h}=k \cdot \gamma_{w}$ where $\gamma_{w}$ is the unit weight of water) and the uniform surcharge loading $q$ applied on the ground surface. Two system responses were considered in the present paper. They are the surface settlement and the consolidation time. The aim of this paper is twofold. Firstly, it presents a global sensitivity analysis based on Sobol indices in order to provide the contribution of each random variable in the variability of the system response. Secondly, it presents a parametric study which aims at investigating the effect of the geotechnical parameters (i.e. drainage condition, degree of consolidation and Biot coefficient) on the PDFs of the system responses. The

*Corresponding author. Email: abed.soubra@univ-nantes.fr 
parametric study also investigates the effect of the statistical parameters (i.e. the coefficient of variation of the random variables and the correlation between these variables) on the PDFs of the system responses.

This paper is organised as follows: First, a brief review of Biot consolidation theory is presented. This is followed by a brief presentation of the CSRSM. Then, the deterministic and the probabilistic analyses and the corresponding numerical results are presented and discussed. The paper ends with a conclusion of the main findings of the paper.

\section{Review of Biot consolidation theory}

The Biot theory of consolidation (Biot 1941, 1956) takes into account the coupling between the water and the soil skeleton. The coupling in FLAC ${ }^{3 \mathrm{D}}$ occurs in two directions: pore-pressure changes cause volumetric strains to occur that influence the stresses. In turn, the pore pressure is affected by the straining that takes place. Notice that the soil matrix is assumed to be homogeneous and it behaves elastically. The pore fluid is coupled to the solid by the conditions of equilibrium and continuity. The one-dimensional consolidation problem in the framework of Biot theory is given by:

$$
\frac{\partial u_{w}}{\partial t}-c \frac{\partial^{2} u_{w}}{\partial z^{2}}=\frac{\alpha}{\alpha_{1} \cdot S} \frac{d \sigma_{z z}}{d t}
$$

where $u_{w}$ and $\sigma_{z z}$ are respectively the excess pore water pressure and the total stress at a spatial location $z$ and at a given time $t, c$ is the coefficient of consolidation, $S$ is the soil elastic storage, $\alpha_{1}$ is a coefficient function of the soil elastic properties and $\alpha$ is the Biot coefficient. The parameters $c, S, \alpha_{1}$ and $\alpha$ are defined as follows:

$$
\begin{gathered}
c=\frac{k}{S} \\
S=\frac{1}{M}+\frac{\alpha^{2}}{\alpha_{1}} \\
\alpha_{1}=\frac{E(1-\nu)}{(1-2 \nu)(1+\nu)} \\
\alpha=1-\frac{K}{K_{s}}
\end{gathered}
$$

In these equations, $k$ is the isotropic permeability coefficient, $M$ is the Biot modulus, $E$ and $v$ are the elastic parameters, $K$ is the drained bulk modulus of the porous material and $K_{s}$ is the bulk modulus of the solid component. It should be remembered here that the permeability coefficient $k$ in Equation (2) is referred to in the literature as the mobility coefficient whose unit is $\left[\mathrm{m}^{2}(\mathrm{~Pa} . \mathrm{s})^{-1}\right]$. It is the coefficient of the pressure term in Darcy's law and it is related to the hydraulic conductivity $k_{h}\left[\mathrm{~ms}^{-1}\right]$ by the expression:

$$
k=\frac{k_{h}}{\gamma_{w}}
$$

Biot modulus $M$ is related to the fluid bulk modulus $K_{f}$ by the following equation:

$$
M=\frac{K_{f}}{n+(\alpha-n)(1-\alpha) \frac{K_{f}}{K}}
$$

where $n$ is the soil porosity. It is generally taken equal to 0.5 . For incompressible grains, $\alpha=1$ and thus $M$ becomes:

$$
M=K_{f} / n
$$

The fluid bulk modulus $K_{f}$ for water is generally taken equal to $2 \times 10^{9} \mathrm{~Pa}$.

In this paper, the stress $\sigma_{z z}$ in Equation (1) is assumed to be constant. Thus, Equation (1) reduces to:

$$
\frac{\partial u_{w}}{\partial t}-c \frac{\partial^{2} u_{w}}{\partial z^{2}}=0
$$

A solution to this equation will enable the excess pore pressure $u_{w}$ to be estimated at any spatial location $z$ and at any time $t$. The vertical displacement $\delta$ is found by the solution of the equilibrium equation together with the mechanical constitutive equation. For incompressible grains (i.e. when $\alpha=1$ ), $M$ tends to infinity and thus, Equation (3) reduces to $\left(S=1 / \alpha_{1}\right)$. Consequently, the consolidation coefficient in Equation (9) becomes:

$$
c=\frac{k E(1-\nu)}{(1-2 \nu)(1+\nu)}
$$

Equation (10) may be re-written as follows:

$$
c=\frac{k}{m_{v}}
$$

where $m_{v}$ is the coefficient of volume compressibility. It is given by:

$$
m_{v}=\frac{(1-2 \nu)(1+\nu)}{E(1-\nu)}
$$

Equation (9) with $\alpha=1$ represents the classical equation of consolidation by Terzaghi. It corresponds to an uncoupled consolidation analysis where the deformation of soil grains is neglected. 


\section{Collocation-based Stochastic Response Surface Method (CSRSM)}

CSRSM is a general and powerful method. It involves the propagation of the uncertainties of the input parameters through a computational model to arrive at a random output vector (Isukapalli et al. 1998, Isukapalli 1999, Phoon and Huang 2007, Huang et al. 2009, Li et al. 2010, Mollon et al. 2011). Thus, this method allows one to determine the PDF of the system response by taking into account the uncertainties of the input parameters via their probability distributions.

The basic idea of the CSRSM is to approximate the system response by a PCE of a suitable order $p$ (i.e. it replaces the complex finite difference or finite element model by a meta-model). This approximation is carried out in the standard space of uncorrelated random variables. Thus, the random input variables (which may be non-normal and/or correlated) should be represented in terms of uncorrelated standard normal random variables. The system response $Y$ may be approximated by $Y_{\text {app }}$ as follows:

$$
Y_{\text {app }}=\sum_{i=0}^{P-1} a_{i} \Psi_{i}(\xi)
$$

where $a_{i}$ are unknown coefficients to be evaluated, $P$ is the size of the expansion (which is equal to the number of the unknown coefficients), $\Psi_{i}$ are multivariate polynomials (chosen herein as the Hermite polynomials) and $\xi$ is a vector of $m$ standard uncorrelated random variables where $m$ is the number of the uncertain parameters. The multivariate Hermite polynomials are given in Isukapalli et al. (1998), Huang et al. (2009) and Mollon et al. (2011) among others. The size $P$ of the PCE is given by:

$$
P=\frac{(m+p) !}{m ! p !}
$$

As may be seen from Equation (13), the output is approximated in the standard space of random variables by a PCE. Its unknown coefficients can be determined either by a regression or by a projection approach. In this work, a regression approach is used. This approach requires the evaluation of the system response at a given number of collocation points. The roots of the one-dimensional Hermite polynomial of order $p+1$ can be used to provide efficient collocation points (Isukapalli et al. 1998, Isukapalli 1999, Phoon and Huang 2007, Huang et al. 2009, Li et al. 2010, Mollon et al. 2011). The collocation points may thus be chosen as the result of all possible combinations of the roots of the one-dimensional Hermite polynomial of $(p+1)$ th order for each random variable.

In this study, the computational deterministic model is a fluid-mechanical interaction model based on FLAC ${ }^{3 \mathrm{D}}$ code. This code is used as a 'black box'. This means that one does not need to modify this code but rather to determine the mechanical response at a given number of collocation points. The determination of the probability distribution of the system response may be summarised by the following steps:

(1) For a prescribed order of the PCE, determine the pattern (number and positions) of the available collocation points in the standard space of random variables. The number $N$ of the available collocation points depends on the number of the random variables $m$ and the chosen PCE order $p$ as follows:

$$
N=(p+1)^{m}
$$

By comparing Equation 14 to Equation15, one can observe that the number of the available collocation points is higher than the number of the unknown coefficients. This leads to a linear system of equations whose number of equations $N$ is greater than the number $P$ of the unknown coefficients. The unknown coefficients of the PCE can be computed using the following equation:

$$
\mathbf{a}=\left(\Psi^{T} \Psi\right)^{-1} \cdot \Psi^{T} . \mathbf{Y}
$$

in which $\mathbf{a}$ is a vector containing the unknown coefficients, $\mathbf{Y}$ is a vector containing the system responses as calculated by the deterministic model at the different collocation points and $\psi$ is a matrix of size $N \times P$ whose elements are the multivariate Hermite polynomials. It is given as follows:

$$
\psi=\left[\begin{array}{llll}
\Psi_{0}^{1}(\xi) & \Psi_{1}^{1}(\xi) & \Psi_{2}^{1}(\xi) \ldots \ldots \ldots \ldots \ldots . . . & \Psi_{P-1}^{1}(\xi) \\
\Psi_{0}^{2}(\xi) & \Psi_{1}^{2}(\xi) & \Psi_{2}^{2}(\xi) \ldots \ldots \ldots \ldots \ldots . . . & \Psi_{P-1}^{2}(\xi) \\
\vdots & \vdots & \vdots & \vdots \\
\vdots & \vdots & \vdots & \vdots \\
\Psi_{0}^{N}(\xi) & \Psi_{1}^{N}(\xi) & \Psi_{2}^{N}(\xi) \ldots \ldots \ldots \ldots \ldots . . \Psi_{P-1}^{N}(\xi)
\end{array}\right]
$$

Several attempts have been made in literature to reduce the number of collocation points by selecting the most efficient points among the available ones (Isukapalli et al. 1998, Isukapalli 1999, Berveiller 2005, Huang et al. 2009, Li et al. 2010). These attempts aim at diminishing the computation time required for the 
probabilistic analyses. The most efficient method is that suggested recently by Sudret (2008). It will be presented later in this section.

(2) Transform the collocation points from the standard to the physical space where the input random variables may be non-normal and/or correlated (see Mollon et al. 2011).

(3) Compute the system response corresponding to each collocation point in the physical space using the deterministic mechanical model.

(4) Determine the values of the unknown coefficients of the PCE using the regression approach by solving the linear system of equations (Equation 16).

(5) Compute the PDF of the approximated system response by performing MCS method on the PCE determined previously and deduce the statistical moments of this distribution.

In order to choose the necessary number of collocation points among the available ones, the method proposed by Sudret (2008) is used herein. It consists in considering the minimal size of the linear system that leads to the invertibility of the information matrix $A$, where, $A=\psi^{T} \psi$. This method can be briefly explained by the following steps: (1) the available collocation points are ordered in a list according to increasing norm, (2) the information matrix $A$ is constructed using the first $P$ collocation points that have the smallest norm, i.e. the ones that are the most close to the origin of the standard space and (3) the information matrix is gradually increased by adding each time the next collocation point from the ordered list. The minimal number of collocation points required to achieve the invertibility of the information matrix is used to compute the PCE coefficients by Equation 16. A quasi-similar approach based on the invertibility of the information matrix was recently used by Li et al. (2010).

Although CSRSM is mainly devoted to the computation of the PDF of the system response, the determination of the PCE in CSRSM has another advantage. The coefficients of the PCE allow one to detect the random variables that have the most significant weight in the variability of the system response using Sobol indices (cf. Sudret 2008, Mollon et al. 2011). For more details on the computation of Sobol indices from the coefficients of a PCE, the reader may refer to Mollon et al. (2011).

\section{Error estimates of the PCE approximation}

The accuracy of a PCE approximation depends mainly on its order. Several procedures can be used to measure the accuracy of a PCE approximation. One of the commonly used procedures is the calculation of the coefficient of determination $R^{2}$. Let us consider $J$ realisations $\left\{\xi^{(1)}=\left(\xi_{1}^{(1)}, \ldots, \xi_{m}^{(1)}\right), \ldots \xi^{(J)}=\left(\xi_{1}^{(J)}, \ldots, \xi_{m}^{(J)}\right)\right\}$ of the standard normal random vector $\xi$, and let $Y=\left\{Y\left(\xi^{(1)}\right), \ldots, Y\left(\xi^{(J)}\right)\right\}$ be the corresponding values of the model response determined by the deterministic calculations. The coefficient of determination $R^{2}$ is calculated as follows:

$$
R^{2}=1-\Delta_{\mathrm{PCE}}
$$

where $\Delta_{\mathrm{PCE}}$ is given by:

$$
\Delta_{\mathrm{PCE}}=\frac{(1 / J) \sum_{i=1}^{J}\left[Y\left(\xi^{(i)}\right)-Y_{\mathrm{app}}\left(\xi^{(i)}\right)\right]^{2}}{\operatorname{Var}(Y)}
$$

and

$$
\begin{gathered}
\operatorname{Var}(Y)=\frac{1}{J-1} \sum_{i=1}^{J}\left[Y\left(\xi^{(i)}\right)-\bar{Y}\right]^{2} \\
\bar{Y}=\frac{1}{J} \sum_{i=1}^{J} Y\left(\xi^{(i)}\right)
\end{gathered}
$$

Notice that $J$ in Equations (19-21) represents the number of collocation points used to evaluate the unknown coefficients of the PCE. The value $R^{2}=1$ indicates a perfect fit of the true model response $Y$, whereas, $R^{2}=0$ indicates a non-linear relationship between the true model $Y$ and the PCE model $Y_{\text {app }}$.

\section{Deterministic analysis}

The deterministic model employed in this paper is based on numerical simulations using the finite

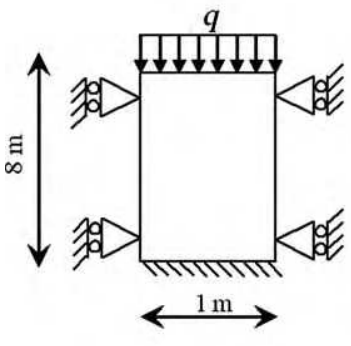

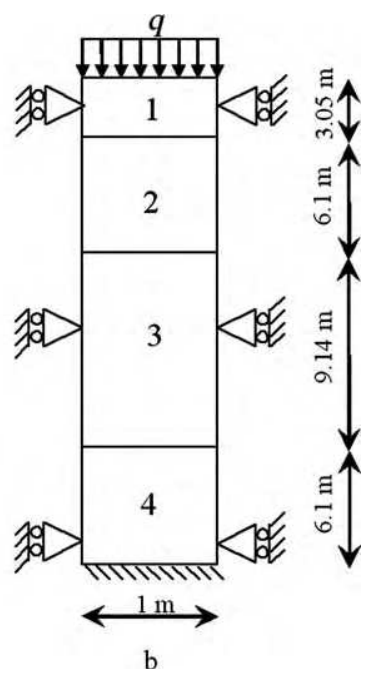

Figure 1. Soil domain for the (a) single clay layer and (b) clay multilayer. 
Table 1. Data used in the single layer and in the four-layer systems shown in Figure 1.

\begin{tabular}{|c|c|c|c|c|}
\hline \multicolumn{5}{|c|}{ Single layer (Badaoui et al. 2007) } \\
\hline$H[\mathrm{~m}]$ & $E[\mathrm{MPa}]$ & $v$ & $k_{h}\left[\mathrm{~ms}^{-1}\right]$ & $q[\mathrm{kPa}]$ \\
\hline 8.0 & 20 & 0 & $10^{-9}$ & 100 \\
\hline \multicolumn{5}{|c|}{ Four-layers (Schiffman and Stein 1970) } \\
\hline Layer number & $H_{i}[\mathrm{~m}]$ & $k_{h i}\left[\mathrm{~ms}^{-1}\right]$ & $m_{v i}\left[\mathrm{kPa}^{-1}\right]$ & $c_{v i}\left[\mathrm{~m}^{2} \mathrm{~s}^{-1}\right]$ \\
\hline 1 & 3.05 & $2.78 \times 10^{-7}$ & $6.41 \times 10^{-5}$ & $4.42 \times 10^{-8}$ \\
\hline 2 & 6.10 & $8.25 \times 10^{-7}$ & $4.08 \times 10^{-5}$ & $2.06 \times 10^{-7}$ \\
\hline 3 & 9.14 & $1.17 \times 10^{-7}$ & $2.04 \times 10^{-5}$ & $5.85 \times 10^{-8}$ \\
\hline 4 & 6.10 & $2.94 \times 10^{-7}$ & $4.08 \times 10^{-5}$ & $7.35 \times 10^{-8}$ \\
\hline
\end{tabular}

difference code FLAC $^{3 \mathrm{D}}$. This code makes use of Biot theory presented previously. Both uncoupled and coupled consolidation analyses were carried out. The computation of the deterministic surface settlement and the corresponding consolidation time and the validation of the obtained results (in both cases of coupled and uncoupled analyses) by comparison with those existing in literature are presented in the two following subsections. This is followed by the
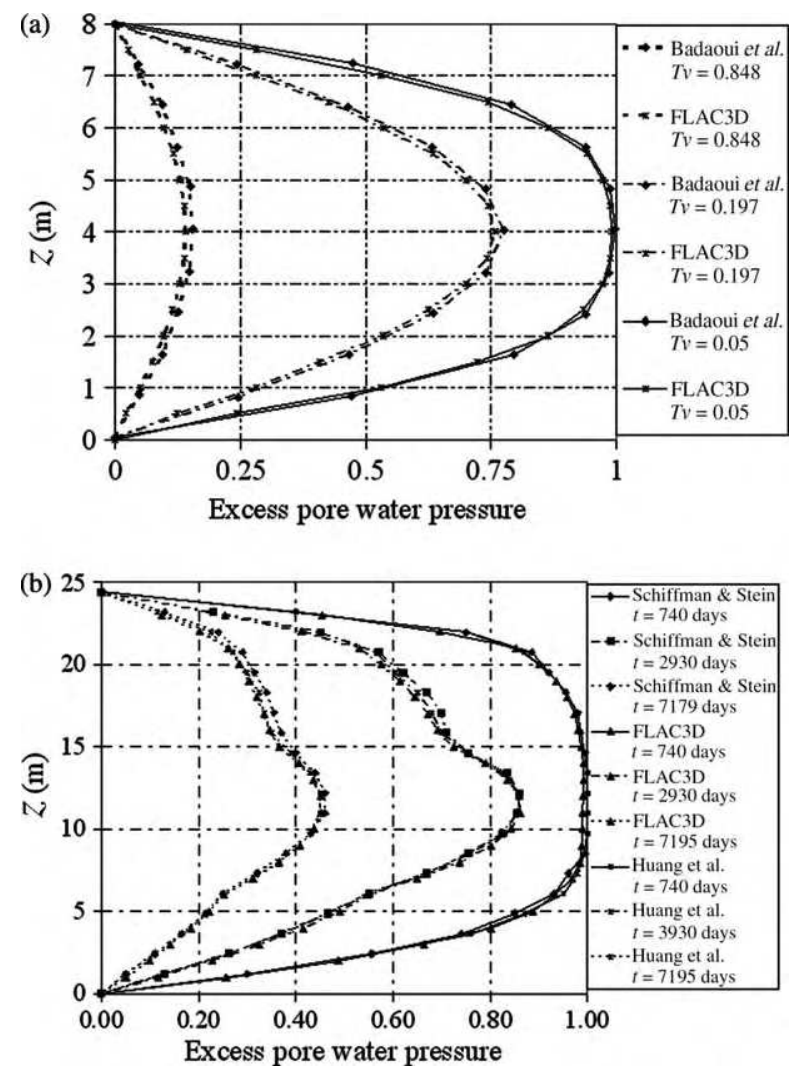

Figure 2. Comparison between present excess pore water pressure distribution and that of other authors for: (a) single clay layer and uncoupled analysis and; (b) clay multilayer and coupled analysis. presentation of a design chart in the case of a single clayey layer considering both coupled $(\alpha<1)$ and uncoupled $(\alpha=1)$ analyses.

\section{Numerical simulations used for the computation of the surface settlement and the corresponding consolidation time (Uncoupled and coupled analyses)}

This section focuses on the computation of the surface settlement and the corresponding consolidation time in both cases of coupled and uncoupled analyses due to a surcharge loading on the ground surface. Two cases of a single layer (Figure 1a) and a multi-layer (Figure 1b) are considered herein. These cases were considered in Badaoui et al. (2007) and Schiffman and Stein (1970), respectively.

For the single saturated clayey layer (cf. Figure 1a and Table 1), a soil mass of unit width and unit length is considered for the consolidation analysis; its thickness is taken equal to $8 \mathrm{~m}$. An uncoupled consolidation analysis with a two-way drainage condition is

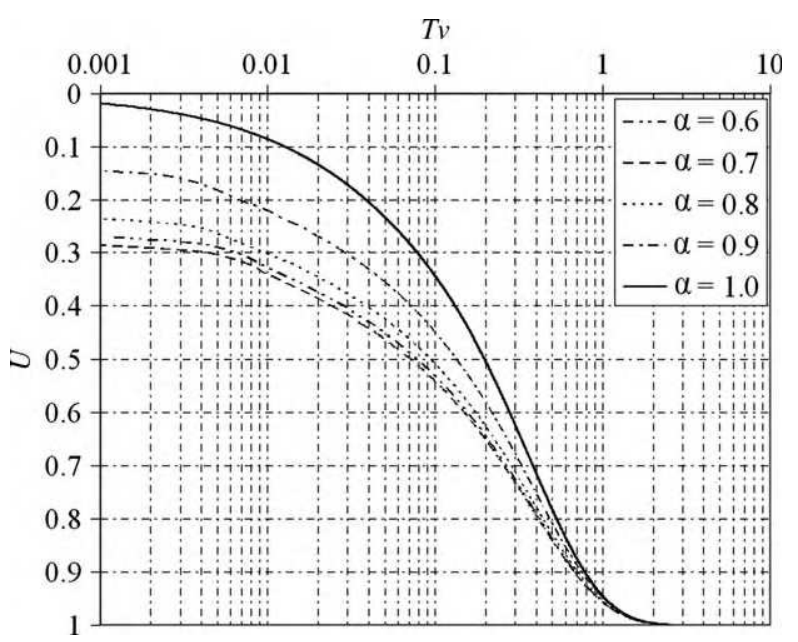

Figure 3. Consolidation degree $U$ vs. time factor $T_{v}$ for different values of Biot coefficient $\alpha$ as given by the present analysis. 
Table 2. Statistical characteristics of the uncertain parameters for the single clayey layer.

\begin{tabular}{lccc}
\hline Parameters & Mean & Coefficient of variation (\%) & Distribution type \\
\hline$E[\mathrm{MPa}]$ & 20 & 20 & Log-normal \\
$v$ & 0.3 & 5 & Log-normal \\
$k_{h}[\mathrm{~m} / \mathrm{s}]$ & $10^{-9}$ & 50 & Log-normal \\
$q[\mathrm{kPa}]$ & 100 & 10 & Log-normal \\
\hline
\end{tabular}

considered in the analysis. The layer is loaded by a uniform surcharge loading $q=100 \mathrm{kPa}$. The soil depth of $8 \mathrm{~m}$ is divided in the vertical direction into 80 equal zones of $0.1 \mathrm{~m}$ each. The bottom boundary was assumed to be fixed and the vertical boundaries were constrained in motion in the horizontal directions.

For the clay multilayer (cf. Figure $1 \mathrm{~b}$ and Table 1), the example initially considered by Schiffman and Stein (1970) and studied recently by Huang et al. (2010) is used. This example involves four compressible layers with free drainage permitted at the top and bottom boundaries. As in the case of a single layer, a soil mass of unit width and unit length is studied. A coupled consolidation analysis is considered herein. A unit uniform surcharge loading is applied at the ground surface. The total depth is divided into zones of $0.1 \mathrm{~m}$ each. The boundary conditions are similar to the case of the single layer.

For both cases, the pressure applied at the ground surface is initially carried by the water, but as time goes on, the water drains through the top and the bottom layer surfaces thus, transferring the load to the soil matrix.

\section{Validation with the existing solutions (Uncoupled and coupled analyses)}

This section aims at validating the deterministic results obtained by the present analysis by comparison with those given by (1) Badaoui et al. (2007) in the case of uncoupled analysis and (2) Schiffman and Stein (1970) and Huang et al. (2010) in the case of a coupled analysis. Figure 2a presents a comparison between the excess pore water pressures obtained by the present model and those provided by Badaoui et al. (2007). However, Figure $2 \mathrm{~b}$ presents the comparison between the results given by the present model and those provided by Schiffman and Stein (1970) and Huang et al. (2010). The comparison was made at three different times after loading. The present coupled and uncoupled results are essentially the same as those presented by the different authors. This validates the present deterministic numerical results. Hence, the deterministic model can be used with confidence in the probabilistic analyses performed in this paper.

\section{Design chart of a single clayey layer for coupled and uncoupled consolidation analyses}

Figure 3 presents a design chart which provides the degree of consolidation $U$ vs. the time factor $T_{v}$ ( where $T_{v}=c t / Z^{2}$ ) for a single layer of depth $=2 Z$ in the case of a two-way drainage condition and for both coupled $(\alpha<1)$ and uncoupled $(\alpha=1)$ consolidation analyses when $n=0.5$. This figure shows that there is a significant decrease in the magnitude of the degree of consolidation between $\alpha=1$ and $\alpha=0.8$. This decrease becomes smaller for smaller $\alpha$-values.

\section{Probabilistic analysis}

This paper aims at performing a probabilistic analysis using CSRSM of a 1D consolidation problem which involves a single clayey layer with a two-way drainage condition. The random variables considered in the analysis are the Young modulus $E$, the Poisson ratio $v$, the hydraulic conductivity $k_{h}$ and the uniform surcharge loading $q$ applied at the ground surface. The illustrative values used for the statistical moments of the different random variables are those commonly

Table 3. Sobol Indices of the maximal surface settlement and the final consolidation time as provided by a fifth order PCE.

\begin{tabular}{lcc}
\hline & \multicolumn{2}{c}{ Values of Sobol indices } \\
\cline { 2 - 3 } Sobol index & Maximal surface settlement & Final consolidation time \\
\hline$S(E)$ & 0.7600 & 0.1425 \\
$S(v)$ & 0.0200 & 0.0082 \\
$S\left(k_{h}\right)$ & $8.89 \times 10^{-7}$ & 0.8492 \\
$S(q)$ & 0.2199 & $1.81 \times 10^{-11}$ \\
Summation & 1.00 & 1.00 \\
\hline
\end{tabular}



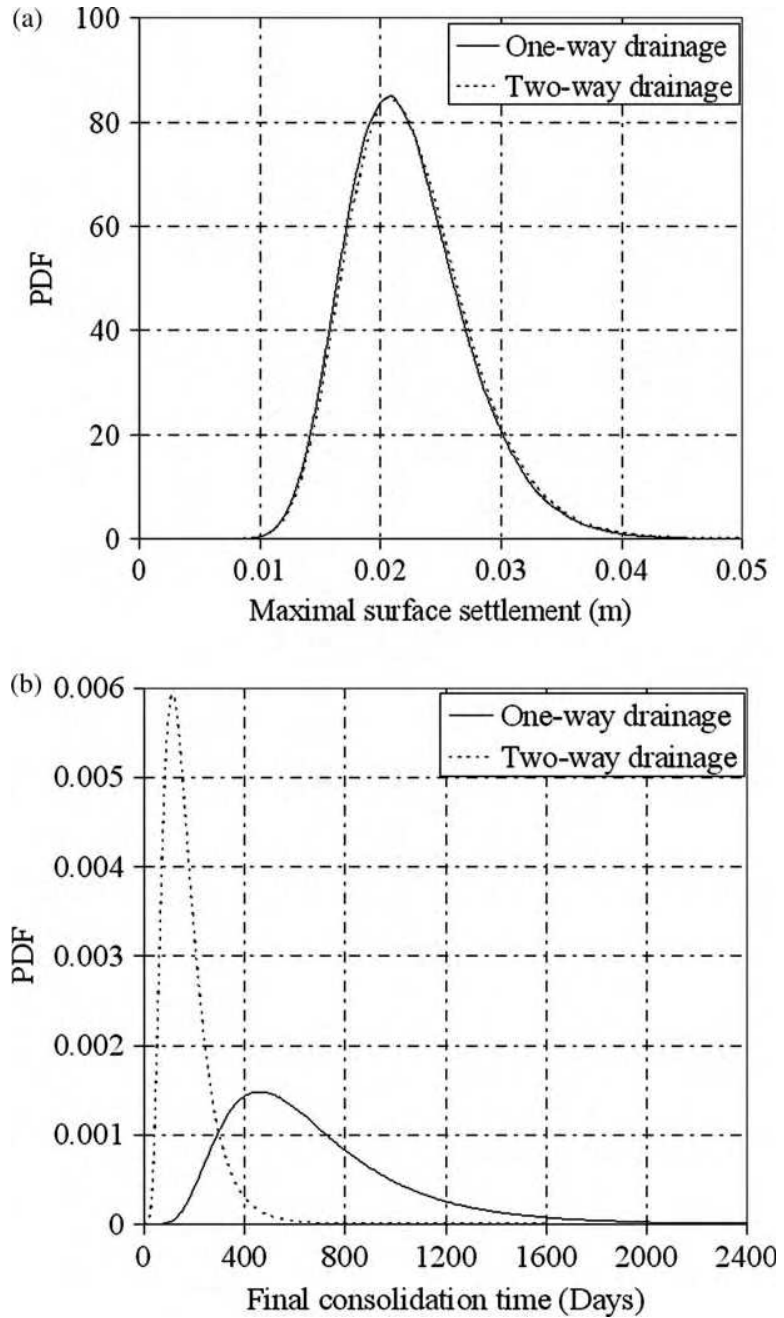

Figure 4. Impact of the drainage conditions on (a) the maximal surface settlement; (b) the final consolidation time when $\alpha=1$.

encountered in practice (Baecher and Christian 2003, Badaoui et al. 2007 among others). These values are called herein the references values. They are given in Table 2. Two system responses were considered hereafter. These are the surface settlement and the corresponding consolidation time for different values of the degree of consolidation. Notice that in this paper, the terms 'maximal surface settlement' and 'final consolidation time' are used for the settlement and the consolidation time corresponding to $U=99.9 \%$.

The consolidation time corresponding to a prescribed value of the degree of consolidation $U$ is computed as follows: The time factor $T_{v}$ corresponding to the prescribed value of $U$ is first determined from Figure 3. This value is then used to compute the consolidation time $t$ as follows: $t=T_{v} \cdot Z^{2} / c$. To assess the corresponding surface settlement, the obtained consolidation time $t$ is used for this computation.

The following subsections focus on the probabilistic results. The optimal PCE order was first determined. Based on the coefficients of the optimal PCE, a global sensitivity analysis based on Sobol indices was performed. Finally, a parametric study that investigates the effect of both the geotechnical input parameters and the statistical characteristics of the random variables on the PDFs of the system responses was presented and discussed.

\section{Optimal PCE order and Sobol indices}

The optimal order of a PCE was determined as the minimal order that leads to a coefficient of determination $R^{2}$ greater than a prescribed value (say 0.9999). The numerical results have shown that a fifth order PCE is necessary for both the maximal surface settlement and the corresponding final consolidation time in order to satisfy this criterion. Thus, this order will be used in all subsequent probabilistic calculations performed in this paper. According to the methodology by Sudret (2008), the number of collocation points required for each PDF computation is equal to 286. This corresponds to a reduction by $78 \%$ in the number of collocation points with respect to the total available collocation points (1297). The computation time when using 286 points is about 390 minutes.

A global sensitivity analysis based on Sobol indices was performed herein to determine the contribution of each random variable in the variability of each system response. The Sobol indices of the different random variables are presented in Table 3 for both the maximal surface settlement and the final

Table 4. Effect of the drainage conditions on the statistical moments of the maximal surface settlement and the final consolidation time.

\begin{tabular}{llccccc}
\hline System response & $\begin{array}{c}\text { Drainage } \\
\text { conditions }\end{array}$ & Mean & $\begin{array}{c}\text { Standard } \\
\text { deviation }\end{array}$ & Skewness & Kurtosis & $\begin{array}{c}\text { Coefficient of variation } \\
(\%)\end{array}$ \\
\hline Maximal surface settlement [m] & One-way & 0.022 & 0.0050 & 0.6916 & 0.8746 & 22.48 \\
& Two-way & 0.022 & 0.0050 & 0.6941 & 0.8784 & 22.40 \\
Final consolidation time [Day] & One-way & 678.98 & 371.29 & 1.7991 & 6.1330 & 54.68 \\
& Two-way & 169.95 & 92.90 & 1.8002 & 6.1851 & 54.67 \\
\hline
\end{tabular}




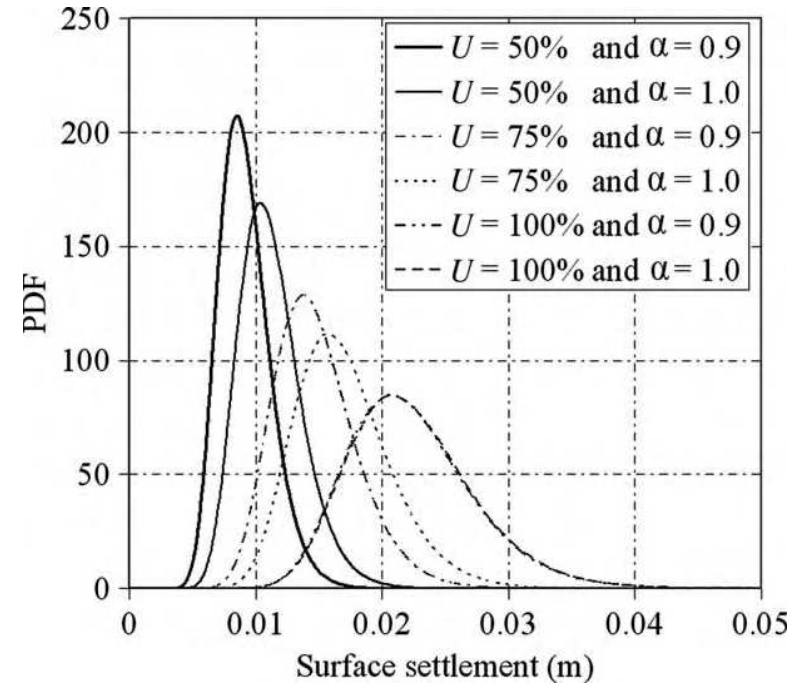

Figure 5. PDFs of the surface settlement for three values of the degree of consolidation and for two values of Biot coefficient $\alpha$.

consolidation time. As expected, the sum of all Sobol indices for each system response is equal to 1 . This table shows that $E$ has a significant contribution in the variability of the maximal surface settlement; however, its contribution in the variability of the final consolidation time is smaller. In contrast, $k_{h}$ has a negligible weight in the variability of the maximal settlement while its weight in the variability of the final consolidation time is significant. Table 3 also shows that the weight of the surcharge loading $q$ in the variability of the maximal surface settlement is relatively large and its weight in the variability of the

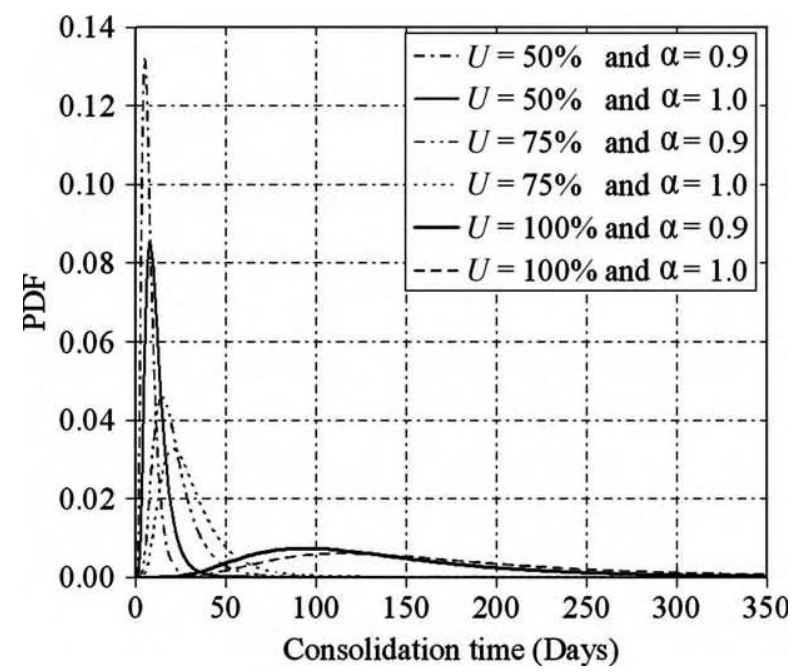

Figure 6. PDFs of the consolidation time for three values of the degree of consolidation and for two values of Biot coefficient $\alpha$. final consolidation time is negligible. Finally, it should be emphasised here that the Sobol indices of $v$ are found to be very small for both system responses. Consequently, only Poisson ratio will be considered hereafter as deterministic for both responses (this is because both system responses are outcome of the same deterministic simulation). On the opposite, the three remaining variables $\left(E, k_{h}\right.$ and $q$ ) will be considered as random variables. Thus, the total number of collocation points that will be used in the subsequent calculations (for a PDF computation) is equal to 82 instead of 286 . The corresponding computation time is about 110 minutes. This reduces again the computation time by $71.3 \%$ with respect to the case where four random variables were used. It should be emphasised herein that the possible reduction in the number of the random variables via the global sensitivity analysis not only reduces the computation time of a probabilistic analysis but also allows to reduce the cost of the experimental investigation of new similar projects because the noninfluential parameters (the Poisson coefficient $v$ in the present paper) do not need a thorough experimental investigation on their variabilities.

\section{Parametric study}

This section aims at presenting a parametric study showing the effect of the geotechnical parameters (i.e. drainage condition, degree of consolidation and Biot coefficient) on the PDFs of the system responses. It also investigates the effect of the statistical parameters of the random variables (i.e. coefficient of variation and coefficient of correlation) on the PDFs of these responses.

\section{Impact of the drainage conditions on the PDFs of the system responses}

Figure 4 shows the effect of the two drainage conditions (one- and two-way) on the PDFs of the maximal surface settlement and the final consolidation time. Only the PDF of the final consolidation time is affected by the drainage conditions; the PDF of the maximal surface settlement being not sensitive to these conditions. Table 4 presents the statistical moments of the PDFs of both responses for the two drainage conditions. Although the standard deviation of the final consolidation time of a one-way drainage condition is much greater than that of the two-way drainage condition, both conditions have the same coefficient of variation for this response. This can be explained by the fact that both drainage conditions consider the same variability for the input variables and thus, the variability of the response 
Table 5. Statistical moments of the surface settlement and the corresponding consolidation time for different values of the degree of consolidation when $\alpha=1$ and $\alpha=0.9$.

\begin{tabular}{|c|c|c|c|c|c|c|c|}
\hline & System response & $\begin{array}{c}\text { Degree of } \\
\text { consolidation }(\%)\end{array}$ & Mean & $\begin{array}{l}\text { Standard } \\
\text { deviation }\end{array}$ & Skewness & Kurtosis & $\begin{array}{l}\text { Coefficient of } \\
\text { variation }(\%)\end{array}$ \\
\hline \multirow[t]{6}{*}{$\alpha=0.9$} & Surface settlement $[\mathrm{m}]$ & 50 & 0.009 & 0.0020 & 0.6871 & 0.8639 & 22.36 \\
\hline & & 75 & 0.015 & 0.0033 & 0.6837 & 0.8474 & 22.36 \\
\hline & & 100 & 0.022 & 0.0050 & 0.6900 & 0.8701 & 22.45 \\
\hline & Consolidation time [Day] & 50 & 7.63 & 4.17 & 1.8198 & 6.5789 & 54.73 \\
\hline & & 75 & 21.96 & 12.00 & 1.7834 & 6.0331 & 54.66 \\
\hline & & 100 & 137.79 & 75.38 & 1.7938 & 6.1351 & 54.71 \\
\hline \multirow[t]{6}{*}{$\alpha=1$} & Surface settlement $[\mathrm{m}]$ & 50 & 0.011 & 0.0025 & 0.6906 & 0.8848 & 22.36 \\
\hline & & 75 & 0.017 & 0.0038 & 0.6871 & 0.8517 & 22.41 \\
\hline & & 100 & 0.022 & 0.0050 & 0.6941 & 0.8784 & 22.40 \\
\hline & Consolidation time [Day] & 50 & 11.85 & 6.47 & 1.7833 & 6.1325 & 54.58 \\
\hline & & 75 & 30.86 & 16.89 & 1.8083 & 6.3080 & 54.74 \\
\hline & & 100 & 169.95 & 92.90 & 1.8002 & 6.1851 & 54.67 \\
\hline
\end{tabular}
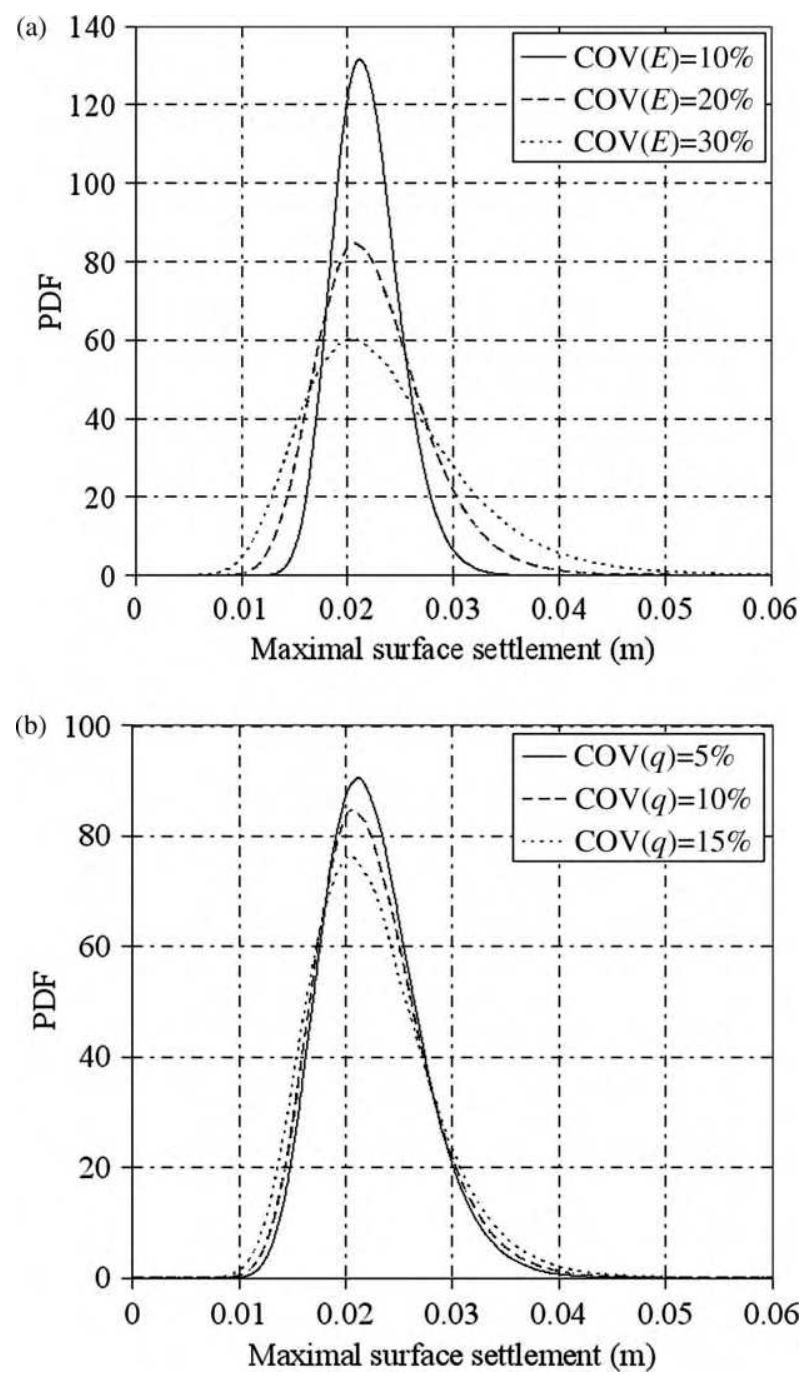

Figure 7. Influence of the coefficients of variation of $E$ and $q$ on the PDFs of the maximal surface settlement when $\alpha=1$ : (a) influence of $\operatorname{COV}(E)$ when $\operatorname{COV}(q)=10 \%$; (b) influence of $\operatorname{COV}(q)$ when $\operatorname{COV}(E)=20 \%$. (when expressed by a non-dimensional parameter) should not be affected by the input variability. The coefficient of variation of the final consolidation time $(\approx 55 \%)$ is much greater than that of the maximal surface settlement $(\approx 22 \%)$. This means that the consolidation time undergoes a significant uncertainty during the propagation of the uncertainties from the input parameters. Therefore, the input uncertain parameters that have a significant weight in the variability of this response (i.e. $k_{h}$ and $E$ ) should be thoroughly investigated in practice. Notice finally that similar to the coefficient of variation, the skewness and kurtosis of both responses are not affected by the drainage conditions. The same explanation given above remains valid herein.

\section{Impact of the degree of consolidation and Biot coefficient on the PDFs of the system responses}

The PDFs of the surface settlement and the corresponding consolidation time for three values of the degree of consolidation $(U=50 \%, 75 \%$ and $100 \%)$ and for two cases of coupled $(\alpha=0.9)$ and uncoupled $(\alpha=1)$ analyses were presented in Figures 5 and 6. The statistical moments corresponding to these PDFs are given in Table 5. From this table, one can observe that the mean value and the standard deviation of both system responses increase with the increase in the degree of consolidation and in Biot coefficient. However, the coefficient of variation, the skewness and the kurtosis of both system responses are almost not affected by the consolidation degree and the Biot coefficient. This is to be expected since the uncertainties of the input parameters remain constant for the different values of $U$ and $\alpha$. 

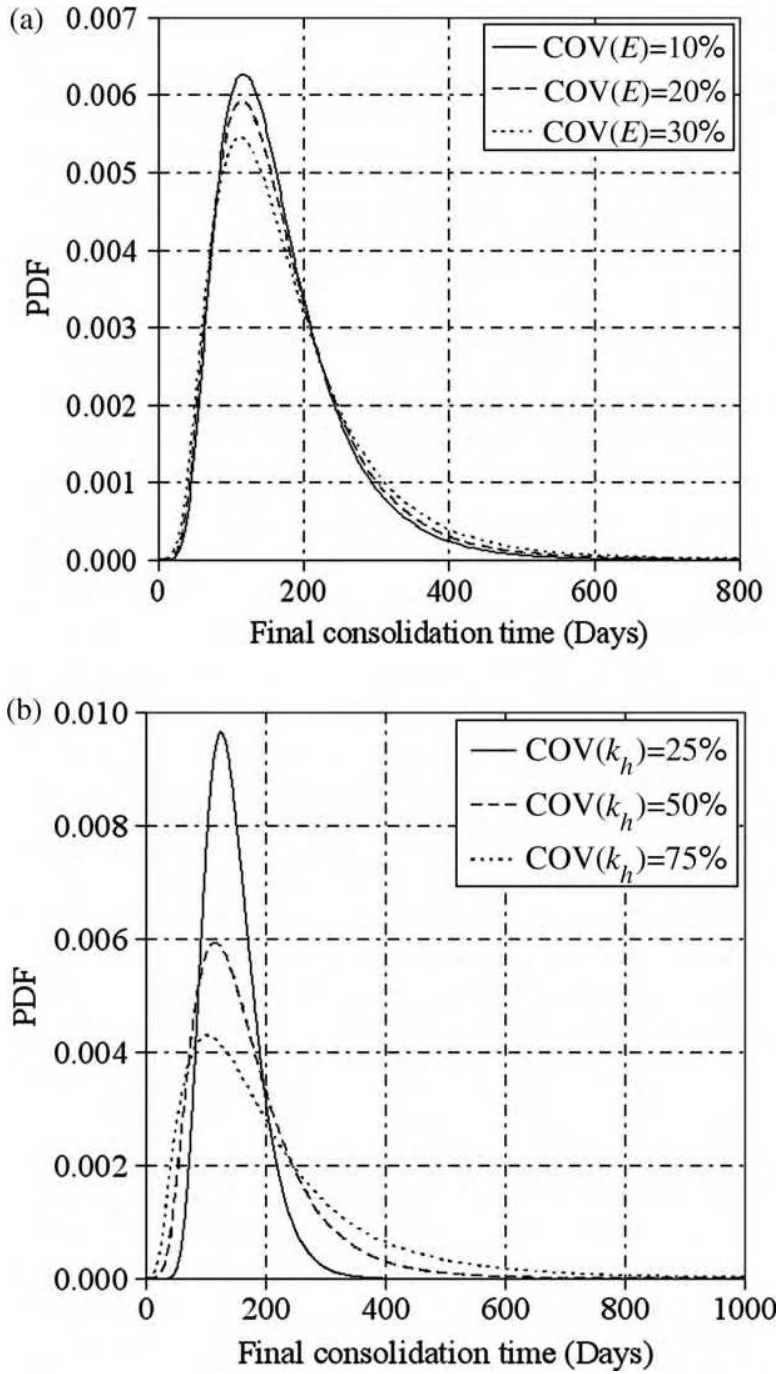

Figure 8. Influence of the coefficients of variation of $E$ and $k_{h}$ on the PDFs of the final consolidation time when $\alpha=1$ : (a) influence of $\operatorname{COV}(E)$ when $\operatorname{COV}\left(k_{h}\right)=50 \%$; (b) influence of $\operatorname{COV}\left(k_{h}\right)$ when $\operatorname{COV}(E)=20 \%$.

Impact of the coefficients of variation (COV) of the different random variables on the PDFs of the system responses

In this section, the effect of $\mathrm{COV}$ of the random variables on the PDFs of the system responses was studied. To investigate the effect of COV of a certain random variable on the PDF of a system response, the $\mathrm{COV}$ of this variable is increased or decreased by $50 \%$ with respect to its reference value given in Table 2; however, the COVs of the other random variables are assumed to be constant (i.e. equal to their reference values).

Figure 7(a), (b) show the impact of the coefficient of variation of $E$ and $q$ on the PDFs of the maximal surface settlement and Figure 8(a), (b) show the impact of the coefficient of variation of $E$ and $k_{h}$ on the PDFs of the final consolidation time. The statistical moments corresponding to these PDFs are given in Tables 6 and 7, respectively. It should be mentioned here that the effect of $\mathrm{COV}_{k_{h}}$ on the maximal surface settlement was not considered because of the negligible contribution of $k_{h}$ in the variability of this response. Similarly, the effect of $\mathrm{COV}_{q}$ on the final consolidation time was not considered because of the negligible weight of $\mathrm{q}$ in the variability of the final consolidation time as was seen previously in the global sensitivity analysis.

Concerning the maximal surface settlement (Table 6), the standard deviation, skewness and kurtosis of this response increase with the increase of $\mathrm{COV}_{E}$ and $\mathrm{COV}_{q}$; however, the mean value of this response remains quite constant. When increasing $\mathrm{COV}_{E}$ and $\mathrm{COV}_{q}$ by $50 \%$ of their reference values, the COV of the maximal surface settlement increases by $41.8 \%$ and $12.4 \%$, respectively. On the other hand, the mean of the final consolidation time increases with the increase in $\mathrm{COV}_{E}$ and $\mathrm{COV}_{k_{h}}$ (Table 7). The increase in $\mathrm{COV}_{E}$ and $\mathrm{COV}_{k_{h}}$ also increases the standard deviation, the skewness and the kurtosis of this response. The COV of the final consolidation time increases respectively by $9.6 \%$ and $44.4 \%$ when increasing $\mathrm{COV}_{E}$ and $\mathrm{COV}_{k_{h}}$ by $50 \%$ with respect to their reference values. Notice finally that the skewness and kurtosis of the maximal surface settlement indicate that its PDF is not far from the Gaussian distribution. In contrast, the skewness and kurtosis of the final consolidation time indicate that its PDF is very far from the Gaussian distribution. As

Table 6. Effect of the coefficients of variation of $E$ and $q$ on the statistical moments of the maximal surface settlement.

\begin{tabular}{ccccccc}
\hline & $\begin{array}{c}\text { Coefficient of } \\
\text { variation }(\%)\end{array}$ & Mean [m] & $\begin{array}{c}\text { Standard } \\
\text { deviation [m] }\end{array}$ & Skewness & $\begin{array}{c}\text { Coefficient of } \\
\text { Kurtosis } \\
\text { variation }(\%)\end{array}$ \\
\hline $\operatorname{COV}(E)$ & 10 & 0.022 & 0.0031 & 0.4332 & 0.3370 & 14.18 \\
& 20 & 0.023 & 0.0050 & 0.6941 & 0.8784 & 22.40 \\
$\operatorname{COV}(q)$ & 30 & 0.024 & 0.0075 & 0.9871 & 1.7886 & 31.77 \\
& 5 & 0.022 & 0.0046 & 0.6324 & 0.7521 & 20.63 \\
& 10 & 0.022 & 0.0050 & 0.6941 & 0.8784 & 22.40 \\
\hline
\end{tabular}


Table 7. Effect of the coefficients of variation of $E$ and $k_{h}$ on the statistical moments of the final consolidation time.

\begin{tabular}{ccccccc}
\hline & $\begin{array}{c}\text { Coefficient of variation } \\
(\%)\end{array}$ & $\begin{array}{c}\text { Mean } \\
{[\text { Day }]}\end{array}$ & $\begin{array}{c}\text { Standard deviation } \\
{[\text { Day }}\end{array}$ & Skewness & Kurtosis & $\begin{array}{c}\text { Coefficient of variation } \\
(\%)\end{array}$ \\
\hline $\operatorname{COV}(E)$ & 10 & 164.99 & 84.54 & 1.6718 & 5.3169 & 51.24 \\
& 20 & 169.95 & 92.90 & 1.8002 & 6.1851 & 54.67 \\
$\operatorname{COV}\left(k_{h}\right)$ & 30 & 177.92 & 106.60 & 1.9859 & 7.6720 & 59.91 \\
& 25 & 144.38 & 46.63 & 1.0006 & 1.8283 & 32.30 \\
& 50 & 169.95 & 92.90 & 1.8002 & 6.1851 & 54.67 \\
\end{tabular}

a conclusion, the mean value of the maximal surface settlement is not affected by $\mathrm{COV}_{E}$ and $\mathrm{COV}_{q}$; however, the mean value of the final consolidation time is sensitive to $\mathrm{COV}_{q}$ and $\mathrm{COV}_{k}$. The variability of the maximal surface settlement is sensitive to $\mathrm{COV}_{E}$ while that of the final consolidation time is sensitive to $\mathrm{COV}_{k_{h}}$. From these results, one can observe that the input parameters for which the COVs are of most significant influence on the variability of a system response are the same as those which have the largest contribution in the variability of this system response (as obtained using Sobol indices).

Table 8 presents the effect of $\mathrm{COV}$ of the most influential input random variables on Sobol indices of the different random variables as computed analytically from the PCE coefficients for both system responses. From this table, one can observe that an increase in the coefficient of variation of a given random variable increases its Sobol index and decreases the Sobol indices of the other random variables. This means that the weight of this random variable in the variability of the system response increases. This automatically reduces the weights of the remaining random variables.
Impact of the correlation between the soil properties ( $E$ and $k_{h}$ ) on the PDFs of the system responses

Figures 9 and 10 show the influence of the correlation coefficient between $E$ and $k_{h}$ on the PDFs of the maximal surface settlement and the final consolidation time. From these figures, one can observe that the correlation between $E$ and $k_{h}$ has a great impact on the PDF of the final consolidation time; however, it has no effect on the PDF of the maximal surface settlement. This may be explained by the negligible contribution of $k_{h}$ in the variability of the maximal surface settlement as was shown before.

Table 9 provides the statistical moments of the final consolidation time for different values of the correlation coefficient. This table shows that the negative correlation reduces the variability of the final consolidation time. Also, it reduces the skewness and kurtosis (i.e. the PDF tends to be Gaussian).

\section{Conclusion}

This paper was devoted to the probabilistic analysis of coupled and uncoupled one-dimensional consolidation problems. A single saturated clayey layer with two situations of one- and two-way drainage

Table 8. Effect of the coefficients of variation of the most influential parameters on Sobol Indices of the different random variables for both responses.

\begin{tabular}{|c|c|c|c|c|c|}
\hline \multirow[b]{2}{*}{ System response } & \multirow{2}{*}{\multicolumn{2}{|c|}{ Coefficient of variation $(\%)$}} & \multicolumn{3}{|c|}{ Sobol indices } \\
\hline & & & $S(E)$ & $S(q)$ & $S\left(k_{h}\right)$ \\
\hline \multirow{6}{*}{$\begin{array}{l}\text { Maximal surface } \\
\text { settlement }[\mathrm{m}]\end{array}$} & \multirow[t]{3}{*}{$\operatorname{COV}(E)$} & 10 & 0.5001 & 0.4998 & $1.2 \times 10^{-4}$ \\
\hline & & 20 & 0.7909 & 0.2090 & $1.0 \times 10^{-4}$ \\
\hline & & 30 & 0.8917 & 0.1082 & $9.0 \times 10^{-5}$ \\
\hline & \multirow[t]{3}{*}{$\operatorname{COV}(q)$} & 5 & 0.9391 & 0.0609 & $4.0 \times 10^{-5}$ \\
\hline & & 10 & 0.7909 & 0.2090 & $1.3 \times 10^{-4}$ \\
\hline & & 15 & 0.6328 & 0.3672 & $2.0 \times 10^{-5}$ \\
\hline \multirow{6}{*}{$\begin{array}{l}\text { Final consolidation } \\
\text { time [Day] }\end{array}$} & \multirow[t]{3}{*}{$\operatorname{COV}(E)$} & 10 & 0.0377 & $6.0 \times 10^{-5}$ & 0.9622 \\
\hline & & 20 & 0.1474 & $5.0 \times 10^{-5}$ & 0.8526 \\
\hline & & 30 & 0.2516 & $4.0 \times 10^{-5}$ & 0.7484 \\
\hline & \multirow[t]{3}{*}{$\operatorname{COV}\left(k_{h}\right)$} & 25 & 0.3875 & $4.0 \times 10^{-5}$ & 0.6125 \\
\hline & & 50 & 0.1474 & $3.0 \times 10^{-5}$ & 0.8526 \\
\hline & & 75 & 0.0531 & $2.0 \times 10^{-5}$ & 0.9469 \\
\hline
\end{tabular}




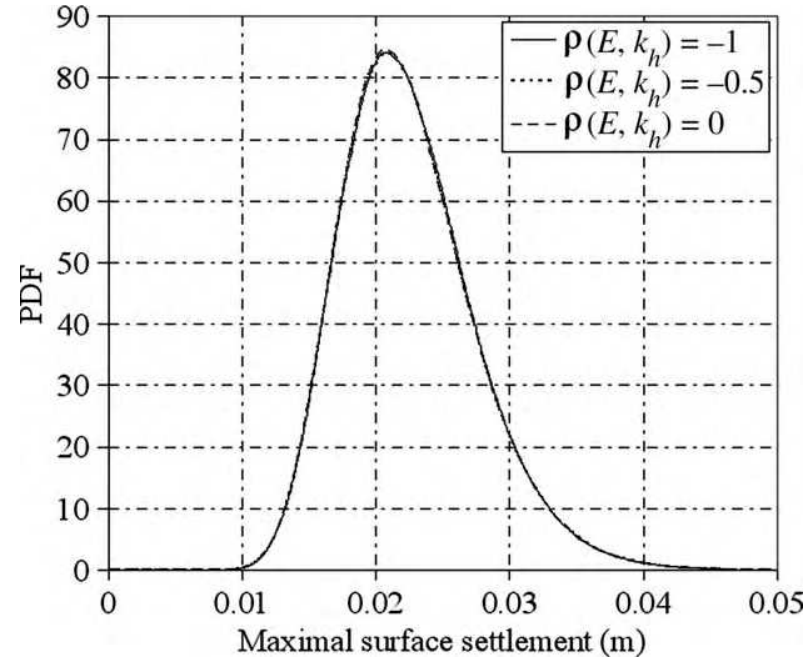

Figure 9. Impact of the correlation coefficient between $E$ and $k_{h}$ on the maximal surface settlement when $\alpha=1$.

conditions was considered in the analysis. The CSRSM was used for the probabilistic analysis. The random variables were the soil Young modulus $E$, the soil Poisson ratio $v$, the soil hydraulic conductivity $k_{h}$ and the uniform surcharge loading $q$ applied at the ground surface. The probabilistic system responses considered in the analysis were the surface settlement and the consolidation time. The deterministic model was based on Biot theory implemented in FLAC ${ }^{3 \mathrm{D}}$ software. The main conclusions of the paper can be summarised as follows:

(1) A design chart $\left(U, T_{v}\right)$ considering both cases of coupled $(\alpha<1)$ and uncoupled $(\alpha=1)$ analyses was presented for practical use in geotechnical engineering. This chart has shown a significant decrease in the magnitude of the degree of consolidation between $\alpha=1$ and $\alpha=0.9$. This decrease becomes smaller for smaller $\alpha$-values.

(2) A global sensitivity analysis based on Sobol indices has shown that the variability of the maximal surface settlement was mainly induced by $E$ and $q$ and that of the final consolidation time was mainly induced by $E$ and $k_{h}$. Poisson ratio has a negligible weight in the variability of

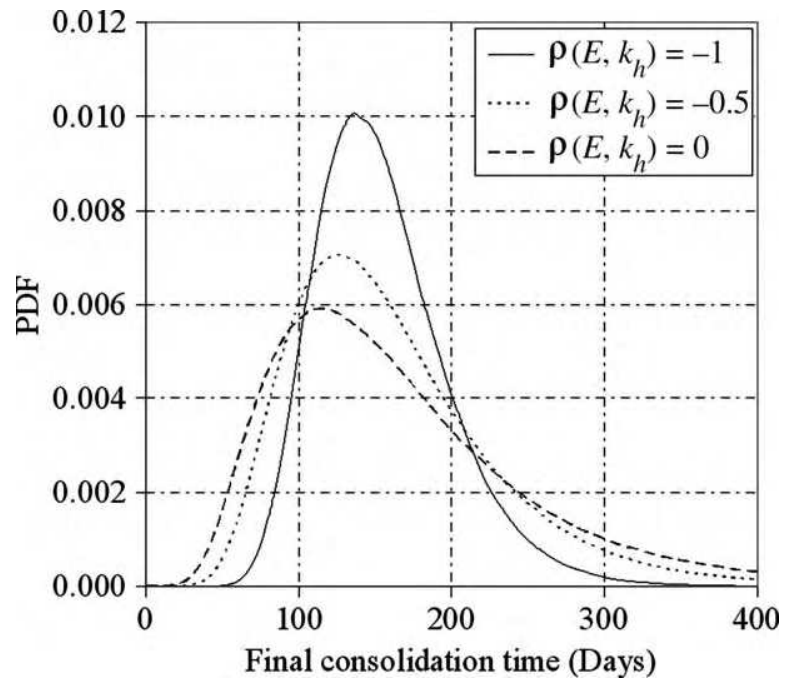

Figure 10. Impact of the correlation coefficient between $E$ and $k_{h}$ on the final consolidation time when $\alpha=1$.

both responses and thus this parameter was considered as deterministic. This strategy of detecting the most influential parameters on the variability of the system responses significantly reduces the computation time of the probabilistic analysis. It has also the advantage of reducing the cost of the experimental investigation of new similar projects because the non-influential parameters do not need a thorough experimental investigation on their variabilities.

(3) While the statistical moments of the maximal surface settlement were found not sensitive to the drainage conditions, the mean and the standard deviation of the final consolidation time were found to be the greatest in the case of the one-way drainage with respect to the case of the two-way drainage, with no change in the value of the coefficient of variation between the two drainage conditions.

(4) The coefficient of variation of the final consolidation time was found much greater than that of the maximal surface settlement. Therefore, the input uncertain parameters that have a significant weight in the variability of this response (i.e. $\mathrm{k}_{h}$ and $E$ ) should be thoroughly investigated in practice.

Table 9. Impact of the correlation coefficient between $E$ and $k_{h}$ on the final consolidation time.

\begin{tabular}{lccccc}
\hline$\rho\left(E, k_{h}\right)$ & Mean [Day] & $\begin{array}{c}\text { Standard deviation } \\
{[\text { Day] }}\end{array}$ & Skewness & Kurtosis & $\begin{array}{c}\text { Coefficient of } \\
\text { variation }(\%)\end{array}$ \\
\hline-1.0 & 154.77 & 43.58 & 0.8740 & 1.4214 & 28.16 \\
-0.5 & 162.18 & 69.57 & 1.3604 & 3.4209 & 42.90 \\
0.0 & 169.85 & 92.99 & 1.8187 & 6.5325 & 54.75 \\
\hline
\end{tabular}


(5) The mean value and the standard deviation of the two system responses increase with the increase in the degree of consolidation and in Biot coefficient. However, the coefficient of variation, the skewness and the kurtosis of both system responses are not affected by neither the consolidation degree nor the Biot coefficient.

(6) The standard deviation, skewness and kurtosis of the two system responses increase with the increase of the coefficients of variation of the input random variables. Concerning the mean value, it remains quite constant for the maximal surface settlement and it increases in the case of the final consolidation time. The coefficient of variation of $k_{h}$ has a more significant effect than that of $E$ on the variability of the final consolidation time. On the other hand, the coefficient of variation of $E$ has a more significant effect than that of $q$ on the variability of the maximal surface settlement.

(7) The input parameters for which the COVs are of most significant influence on the variability of a system response are the same as those which have the largest contribution in the variability of this system response (as obtained using Sobol indices).

(8) An increase in the coefficient of variation of a given random variable increases its Sobol index. This means that the weight of this random variable in the variability of the system response increases. This automatically reduces the weight of the remaining random variables.

(9) The correlation between the variables $E$ and $k_{h}$ has a considerable effect on the final consolidation time; however, it has no effect on the maximal surface settlement. The negative correlation was found to reduce the variability of the final consolidation time. It also leads to smaller values of the skewness and kurtosis.

\section{References}

Badaoui, M., Nour, A., Slimani, A., and Berrah, M.K., 2007. Consolidation statistics investigation via thin layer method analysis. Transport in Porous Media, 67, 69-91.

Baecher, G.B. and Christian, J.T., 2003. Reliability and statistics in geotechnical engineering. London and New York: John Wiley.

Bauer, J. and Pula, W., 2000. Reliability with respect to settlement limit-states of shallow foundations on linearly-deformable subsoil. Computers and Geotechnics, 26, 281-308.

Berveiller, M., 2005. Eléments finis stochastiques: approches intrusives et non intrusives pour des analyses de fiabilité.
[Stochastic finite elements: intrusive and non-intrusive approaches for reliability analysis] Thesis (PhD). Université Blaise Pascal-Clermont Ferrand, France. [In French].

Biot, M.A., 1941. General theory of three-dimensional consolidation. Journal of Applied Physics, 12 (2), 155-164.

Biot, M.A., 1956. General solutions of the equations of elasticity and consolidation for a porous material. Journal of Applied Mechanics, Transactions, ASME, 78, 91-96.

Chang, C.S., 1985. Uncertainty of one-dimensional consolidation analysis. Journal of Geotechnical Engineering, 111 (12), 1411-1424.

Darrag, A.A. and Tawil, M.A., 1993. The consolidation of soils under stochastic initial excess pore pressure. Applied Mathematical Modelling, 17, 609-612.

Freeze, R.A., 1977. Probabilistic one-dimensional consolidation. Journal of the Geotechnical Engineering division, ASCE, 103 (GT7), 725-741.

Griffiths, D.V. and Fenton, G.A., 2009. Probabilistic settlement analysis by stochastic and random finiteelement methods. Journal of Geotechnical and Geoenvironmental Engineering, ASCE, 135 (11), 1629-1637.

Hong, H.P., 1992. One-dimensional consolidation with uncertain properties. Canadian Geotechnical Journal, $29,161-165$.

Huang, J., Griffiths, D.V., and Fenton, G.A., 2010. Probabilistic analysis of coupled soil consolidation. Journal of Geotechnical and Geoenvironmental Engineering, ASCE, 136 (3), 417-430.

Huang, S.P., Liang, B., and Phoon, K.K., 2009. Geotechnical probabilistic analysis by collocation-based stochastic response surface method: an EXCEL add-in implementation. Georisk, 3 (2), 75-86.

Hwang, D. and Witczak, M.W., 1984. Multidimensional probabilistic consolidation. Journal of Geotechnical Engineering, ASCE, 110 (8), 1059-1077.

Isukapalli, S.S., 1999. Uncertainty analysis of transporttransformation models. Thesis (PhD). The State University of New Jersey.

Isukapalli, S.S., Roy, A., and Georgopoulos, P.G., 1998. Stochastic response surface methods (SRSM) for uncertainty propagation: application to environmental and biological Systems. Risk Analysis, 18 (3), 357-363.

Lee, P.K.K., Xie, K.H., and Cheung, Y.K., 1992. A study on one-dimensional consolidation of layered systems. International Journal for Numerical and Analytical Methods in Geomechanics, 16 (11), 815-831.

Li, D., Chen, Y., Lu, W., and Zhou, C., 2010. Stochastic response surface method for reliability analysis of rock slopes involving correlated non-normal variables. Computers and Geotechnics, 38 (1), 58-68.

Mollon, G., Dias, D., and Soubra, A.-H., 2011. Probabilistic analysis of pressurized tunnels against face stability using collocation-based stochastic response 
surface method. Journal of Geotechnical and Geoenvironmental Engineering, ASCE, 137 (4), 385-397.

Nour, A., Slimani, A., and Laouami, N., 2002. Foundation settlement statistics via finite element analysis. Computers and Geotechnics, 29, 241-672.

Phoon, K.K. and Huang, S.P., 2007. Geotechnical probabilistic analysis using collocation-based stochastic response surface method. In: Jun Kanda, Tsuyoshi Takada \& Hitoshi Furuta, ed. Proc., Applications of statistics and probability in civil engineering. Tokyo: Taylor and Francis, 45-51.

Schiffman, R.L. and Stein, J.R., 1970. One-dimensional consolidation of layered systems. Journal of Soil Mechanics and Foundations Division, 96, 1499-1504.

Sudret, B., 2008. Global sensitivity analysis using polynomial chaos expansion. Reliability Engineering And System Safety, 93, 964-979. 